\title{
Can Free Electron Lasers Answer Critical Questions in Ultraviolet Photobiology?
}

\author{
John C. Sutherland \\ Biology Department and the National Synchrotron Light Source \\ Brookhaven National Laboratory, Upton, NY 11973-5000
}

\begin{abstract}
DNA and other biological macromolecules are sensitive to damage by ultraviolet light with wavelengths less than about 320 $\mathrm{nm}$. Fortunately, such wavelengths are efficiently absorbed by ozone in the stratosphere. DNA can also be damaged by longer wavelength ultraviolet and (perhaps) visible light, albeit with far lower efficiencies. Indeed, the cross section for damage induction in DNA by longer wavelengths is so low that it has been difficult to measure easily in controlled experiments using monochromatic laboratory sources. However, the flux of solar photons reaching the biosphere is far higher than at the shorter wavelengths. Thus the damage induced by wavelengths $>320 \mathrm{~nm}$ may constitute a significant fraction of the total UV burden in a particular organism or ecosystem. Understanding the nature and extent of this long wavelength induced damage is crucial in several important societal problems including:
\end{abstract}

- Evaluating the biological impact of depletion of stratospheric ozone.

- Determining if sunscreen preparations offer the same protection against long term effects such as cutaneous malignant melanoma as they do against prompt effects such as erythema (sunburn).

- Determining the safest spectrum for use in indoor tanning.

The light sources required for such studies must provide high intensities of nearly monochromatic light that can be tuned to any wavelength in the required spectral range. This paper will evaluate the potential of ultraviolet free electron lasers, and particularly the soon to be available UV-FEL at the Thomas Jefferson National Accelerator Facility for such experiments.

Keywords: free electron lasers, ultraviolet radiation, environmental photobiology, ozone depletion,

\section{INTRODUCTION}

Environmental photobiology is the study of the effects of "light" on the biosphere. "Light" for our purposes includes all wavelengths in the range spanned by spectrum from roughly $200 \mathrm{~nm}$ to $1 \mu \mathrm{m}$, where water is transparent, and hence includes parts of the ultraviolet and near-infrared as well as the visible. Many of the effects of light are beneficial, and indeed necessary for life to exist; photosynthesis and vision are two examples. However, the ultraviolet portion of this spectrum wavelengths $\leqslant 400 \mathrm{~nm}$ - produce a variety of deleterious effects. Recent concern regarding the consequences of enhancing the intensity of the shortest solar wavelengths that reach the surface of the earth as a result of depletion of stratospheric ozone have resulted in interest in understanding the biological effects of such radiations and developing the scientific capabilities required to make realistic predictions regarding their biological impact. Enormous advances have been made in our theoretical understanding of the biological effects of ultraviolet radiation, and the molecular and cellular tools available to study their effects. A significant gap in our experimental capabilities remains, however, in producing high intensity ultraviolet radiation under laboratory conditions for some critical experimental studies. This gap is greatest not at the shortwave limit of the solar spectrum, where the intensity of sunlight is low, but rather at the long-wavelength end of the UV, where the cross-section for producing damage is low, but finite and ambient radiation from the sun is high. This paper discusses several questions in ultraviolet photobiology of societal importance and the properties of free electron lasers that may aid scientists in finding the answers. 


\section{ULTRAVIOLET ENVIRONMENTAL PHOTOBIOLOGY}

\section{DNA is Usually the Principal Target}

Ultraviolet radiation, like ionizing radiation, damages many of the molecular species that make up a cell. However, DNA is usually the critical target in cell killing, and always the molecular species that must be damaged if a mutation is produced. (One of the rare exceptions to the rule that DNA is the critical target for cell killing is provided by certain bacteria that have phenomenal ability to repair damage to their DNA.) The pivotal role of DNA in photo- and radiobiology derives from its function as the information repository for the cell. Damaged proteins, lipids, RNA, ..., can be replaced by new synthesis, but damaged DNA must either be repaired or the cell must live with the damage. Note that mutations can arise from incorrect repair. The central role of DNA is further emphasized by the existence of multiple mechanism, to repair damaged DNA in organisms ranging from bacteria to man.

\section{Action Spectra and Solar Insolation}

Radiation from the sun, and most artificial sources for that matter, consists of a broad spectrum. Thus, we must deal with the fact that DNA is damaged with different efficiencies at different wavelengths. A further complication is that the mixture of photoproducts produced may be different in different portions of the spectrum. (One of the many differences between the damage produced by ultraviolet radiation and ionizing radiation is that, at least for $\lambda \leq \approx 320 \mathrm{~nm}$, ultraviolet induced damage results mostly from direct absorption by DNA, while for ionizing radiation, most damage results from absorption by water, which generates hydroxyl radicals and other reactive species). We calculate the damage of a particular type produced by a broad spectrum by integrating the product of the cross section for producing that particular damage at wavelength $\lambda$ and the intensity of the radiation at that wavelength, as shown in Equation 1.

$$
<\text { effect }_{i}>\int \sigma_{i}(\lambda) I(\lambda) d \lambda . \quad \text { Equation } 1
$$

We can define the parameters in Equation 1 in two different ways. We can think of $\sigma_{i}(\lambda)$ as the absolute cross section for inducing damage of type $i$, and $I(\lambda)$ as the intensity of the radiation actually bathing the target, or we can treat $\sigma_{i}(\lambda)$ as the cross section for damaging the target in situ and $I(\lambda)$ as the intensity of the radiation incident on the target organism, where it is much easier to measure. In the latter case, $\sigma_{i}(\lambda)$ is a property of both the target molecule and its location within a cell or organism. We shall adopt the latter point of view for the data discussed here. In the photobiology community, $\sigma_{i}(\lambda)$ is called the action spectrum for producing damage of the $i^{\text {th }}$ class. $^{\dagger}$

The spectral integrals defined in Equation 1 constitute a measure of the photochemical damage of a particular type produced in DNA, but generally not the resultant biological damage. The ultimate biological effects are linked to the various photochemical damages through functional dependencies that are almost always non-linear. 1 In addition, if there are multiple damages that are repaired in different ways, there may be synergistic interactions between them. Such interactions may arise in damages induced by the solar spectrum because wavelengths less than about 320 nm mainly induce cyclobutyl and 6-4 pyrimidine dimers, which are repaired by nucleotide excision repair while longer ultraviolet wavelengths produce a higher fraction of oxidative damages that are repaired by base excision repair. ${ }^{2}$

\section{The Alphabetical Ultraviolet}

It is frequently convenient to assign names to various regions of the electromagnetic spectrum; microwave, infrared, visible, ultraviolet, X-ray, are examples. Similarly, broad regions are frequently subdivided. In the case of the visible spectrum we use the names of colors, while in the ultraviolet, the physics community has used transmission properties, i.e., quartz and

\footnotetext{
t Action spectra can be either quantitative, as in the example above, or relative, which frequently are useful in describing biological systems that are difficult to describe using quantitative endpoints. In addition, some effects may not respond linearly to radiation, e.g., cell death or mutation induction. In such systems, action spectra are defined as the reciprocal of the dose of radiation required to produce a given level of effect. In such cases, the detailed shape of an action spectrum may change depending on the level of effect chosen for the determination. Erythema is an example of an effect in which only a threshold is measured, so there is no dose response function. Instead, the minimal erythermal dose (MED) is recorded, which is the lowest dose of UV that produces observable erythema, typically measured $24 \mathrm{~h}$ after irradiation. While one can speak of half of the MED or twice the MED, it is difficult to determine a level of erythema that is half or twice that of the level of erythema observed at the threshold level. In the case of erythema, action spectra are usually obtained by plotting the reciprocal of the MED.
} 
vacuum ultraviolet. Photobiologists have adopted an alphabetic scheme for subdividing the ultraviolet, the rationale for which is shown in Figure 1
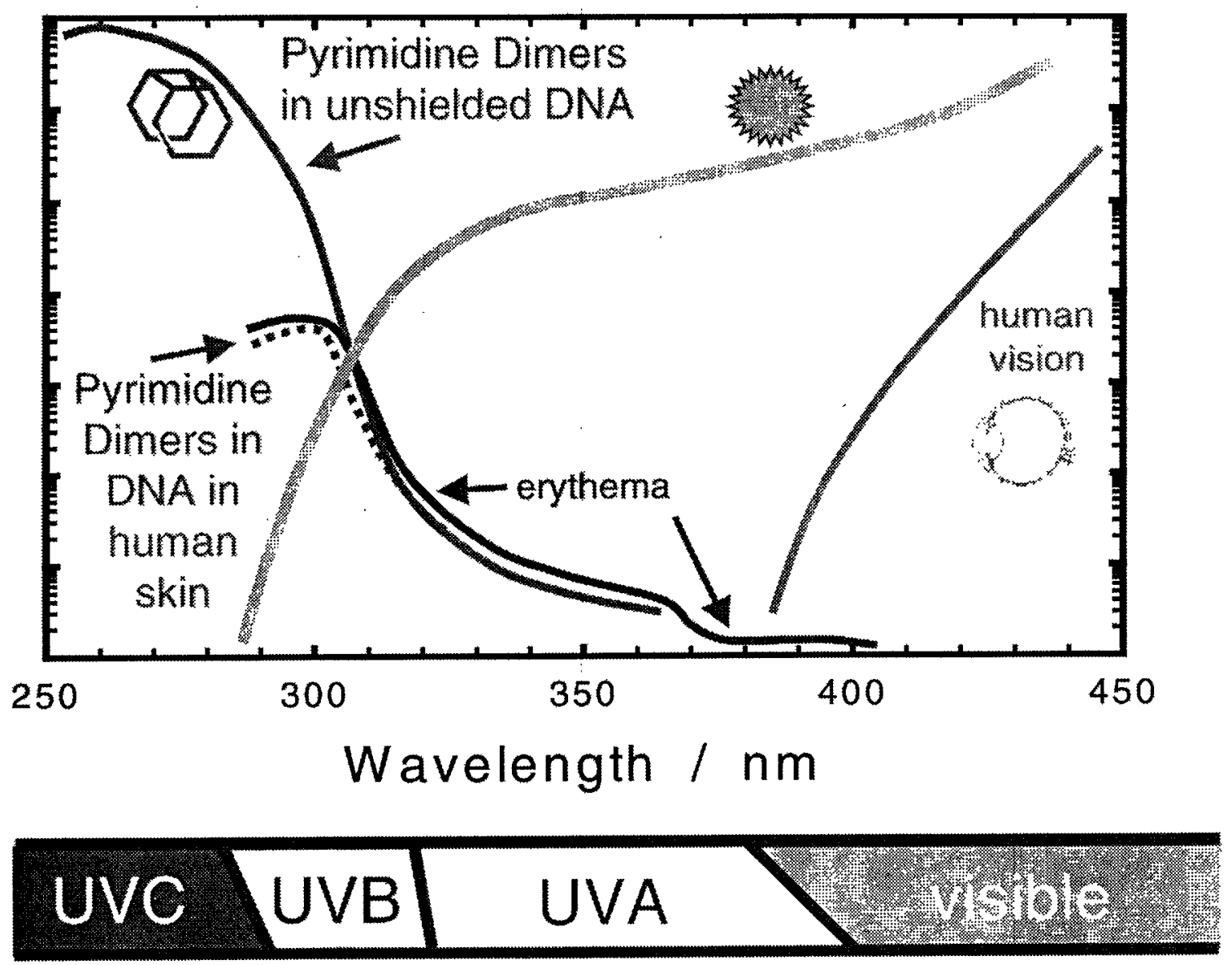

Figure 1 Schematic representations of the action spectra for the induction of DNA damages (pyrimidine dimers measured as endonuclease sensitive sites) in unshielded DNA ${ }^{3}$ and in DNA in human $\operatorname{skin}^{4}$, erythema ${ }^{5}$, human vision and a typical terrestrial mid-day mid-latitude solar spectrum. Note that the vertical scales are logarithmic and span many decades. The ranges of the names given to various subdivisions of the UV are shown below the wavelength axis.

The divisions are based on the solar spectrum that reaches the surface of the earth and on the susceptibility of biological systems to damage - which, as noted above, is the susceptibility of DNA. The UVA is that spectral region in the ultraviolet where sunlight is intense, but the cross-section for damaging DNA is low. The UVB is a transition zone. The intensity of solar radiation drops rapidly (due to absorption by ozone in the stratosphere), while the cross-section for damaging DNA increases rapidly with decreasing wavelength. Finally, at shorter wavelengths, DNA is easily damaged (if unprotected by local absorption such as the upper layers of the skin), but no solar radiation ever reaches the earth's surface. This region is called the UVC, which includes the 254 emission line generated by a low pressure mercury lamp.

Although the division of a continuum into discrete zones is always somewhat arbitrary, the limits of the regions have been defined by international convention. The UVA is defined as extending from 320 to $400 \mathrm{~nm}$ (although human vision in normal people extends to about $380 \mathrm{~nm}$ if careful measurements are made). The UVB is defined as extending from 290 to 320 and the UVC is for wavelengths less that $290 \mathrm{~nm}$ (the lower limit is unspecified). These divisions are summarized in Table 1. In the past, the UVA/UVB boundary sometimes has been designated as $315 \mathrm{~nm}$ and the UVB/UVC boundary as 
$280 \mathrm{~nm}$. My view is that the exact values are less important than the underlying concepts, and that the boundaries should properly be regarded as somewhat vague.

Table 1 Definitions of the divisions in the ultraviolet used in photobiology

\begin{tabular}{cccc}
\hline Name of Region & Wavelength limits $(\mathrm{nm})$ & DNA damage cross section & Terrestrial Solar Intensity \\
\hline UVA & $320-400$ & low & high \\
UVB & $290-320$ & increasing & decreasing \\
UVC & $<290$ & high & none \\
\hline
\end{tabular}

\section{DNA Damage Varies by Spectral Region}

In the UVB and UVC the principal lesions are formed between pyrimidines that are adjacent to one another on the same strand of DNA. The two main types are cyclobutyl and 6-4 dimers and may involve both thymine and cytosine nucleotides in both the 3 ' and 5 ' positions. ${ }^{6}$ In the UVA, a variety of other damages become important, including oxidative damages. Such lesions are similar to some of the classes of damages produced by ionizing radiation. Pyrimidine dimers are bulky lesions that are repaired by nucleotide excision repair, which opens the DNA strand and replaces a number of bases using the bases on the opposite strand as a templet. In addition, cyclobutyl dimers can be repaired by photoreactivation, which reverses the lesion without breaking the phospho-diester backbone of the DNA. In contrast, oxidative damages are usually repaired by base excision repair, which opens the DNA backbone, but replaces fewer bases - sometimes only one nucleotide.

The photophysical and photochemical reactions that produce oxidative damages are different from those that produce dipyrimidines. While pyrimidine dimers can be sensitized by resonant energy transfer, particularly triplet-triplet transfer, they can also be formed by direct absorption of incident UV by DNA, which is usually the dominant mechanism. The photophysics and photochemistry leading to oxidative damage is less well understood, but, in analogy to the induction of DNA damage by ionizing radiation, the involvement of absorbing species other than DNA is a strong possibility. One important difference with ionizing radiation, is that water can absorb X-rays and $\gamma$-rays but not UVA. Another parameter that is wavelength dependent is the distance of penetration into an organism - in general, longer wavelengths penetrate further than shorter wavelengths. For example, UVA can reach the dermas of light skinned humans, while UVB is mainly limited to the epidermis.

\section{Why is the UVA Important?}

The damage induced by UVA radiation has received far less attention than that produced by UVB and UVC, but may be important in several areas.

Depletion of Stratospheric Ozone: The short wavelength limit of solar radiation reaching the surface of the earth is determined by ozone in the stratosphere. Decreases in stratospheric ozone will increase the UVB reaching the biosphere, but all of the increase will occur for wavelengths less than $320 \mathrm{~nm}$. The largest fractional increases occur at the short wavelength end of the UVB. Because ozone depletion will not effect insolation of UVA, it might appear that consideration of UVA effects have no role to play in discussions of ozone depletion. The involvement of the UVA results from the way we describe the effects of ozone depletion as a fractional increase in damage levels. Referring to Equation 1, we can describe the increase in the damage of type $i$ by Equation 2, where $\left\langle\right.$ initial $_{i}>$ and $<$ final $_{i}>$ represents the levels of damage of type $i$ before and after a specific degree of ozone depletion. We can always define the increase in this class of damage according to Equation 3.

$$
\begin{aligned}
& \text { \%increase }_{i}=100\left(\frac{\left.<\text { final }_{i}\right\rangle}{\left.<\text { initial }_{i}\right\rangle}-1\right) \\
& <\text { final }_{1}>=<\text { initial }_{1}>+<\text { increase }_{1}> \\
& \text { \%increase }_{i}=100\left(\frac{\left.<\text { increase }_{i}\right\rangle}{\left\langle\text { initial }_{i}\right\rangle}\right)
\end{aligned}
$$


A simple rearrangement leads to Equation 4, which indicates that underestimating the initial damage level has exactly the same effect as overestimating the increase in the level of damage. Thus, realistic predictions of the effects of ozone depletion require that we allow for damages induced by all regions of the solar spectrum. An obvious complication is that the classes of damage that will be enhanced by the increasing UVB may be quantitatively different in composition than the distribution of lesions in the initial distribution.

Action Spectra for Induction of Skin Cancers: The overwhelming number of human skin cancers are either basal or squamous cell carcinomas, which are rarely fatal. Most human mortality from skin cancers results from cutaneous malignant melanomas. The incidence of cutaneous malignant melanoma has been increasing rapidly in light skinned human populations. ${ }^{7}$ Experimental action spectra for basal cell carcinomas, which have been determined in mice, generally parallel the action spectrum for human erythema shown in Figure 2, although there is some hint that there may be structure at the longer wavelengths. ${ }^{7}$

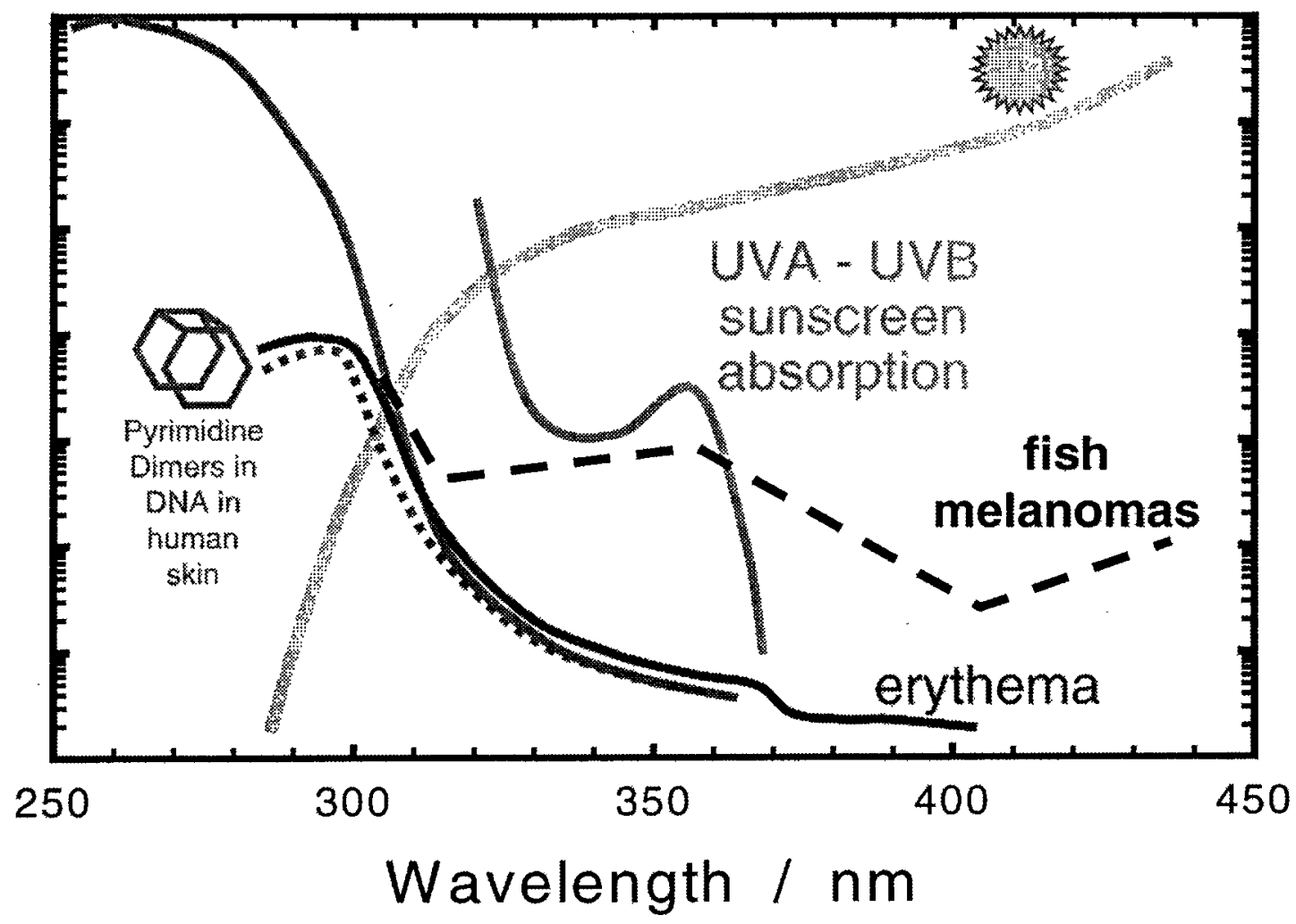

Figure 2 Action spectra for DNA damage in isolated DNA and in the DNA of human skin, erythema, and typical absorption spectra for a UVB/UVA sunscreen, plus the action spectra reported for melanoma induction in a $\mathrm{fish}^{8}$, which decreases at long wavelengths much less slowly than other biological responses.

The action spectrum for cutaneous malignant melanoma in humans is presently unknown, and experimental induction of melanomas in human subjects is obviously not possible for ethical reasons. Unfortunately, murine models for cutaneous malignant melanomas are not presently available. An action spectrum for melanomas in a fish model has been reported ${ }^{8}$ and is sketched in Figure 2. Data are available for only five wavelengths and there appears to be considerable experimental 
scatter, but the salient feature of this action spectrum is that the sharp decrease in cross section with increasing wavelength as observed for pyrimidine dimer induction and human erythema - does not occur. Melanomas arise in melanocytes, and these cells are inherently pigmented so they absorb strongly throughout the UVA, and the visible spectrum. If the action spectrum for induction of cutaneous malignant melanoma in humans is similar to that reported for the fish system, sunscreen preparations will protect more efficiently against erythema than against cutaneous malignant melanoma. Sunscreen use permits extended exposures to solar radiation without induction of immediate erythema to signal an overexposure. The very long delay in cancer induction means - if this scenario is correct - that the widespread use of sunscreens may actually contribute to future increases in incidence of cutaneous malignant melanoma in the years ahead. This possibility is extremely controversial! On one hand, there are some epidemiological ${ }^{9}$ and indirect clinical data ${ }^{10}$ that appear to support this interpretation. On the other hand, concern about the possible use of sunscreens may lead to reduced use of conventional sunscreens by some segments of the population - thereby reducing the established benefits of sunscreen use in preventing non-melanoma skin cancers and photoaging. Nor is it practical to adopt sunscreen products that block all possible wavelengths that may be actinic. Until good action spectra for melanoma induction are available, we don't know what to protect against. In addition, sunscreens that absorb above $400 \mathrm{~nm}$ will be colored, which is less than ideal for products that are marketed much like cosmetics. Thus, there are several compeling reasons why we need to obtain action spectra for melanoma induction in appropriate mammals.

Indoor Tanning: Tanning salons are a $5 \mathrm{G} \$ \mathrm{y}$ industry in the United States. The equipment used for indoor tanning is regulated by the Center for Devices and Radiological Health of the U.S. Food and Drug Administration. Some dermatologists and public advocacy groups have recommended enhanced warning labels or even a ban on such devices. The FDA, along with the National Cancer Institute and Center for Communicable Diseases organized a workshop on the "Risks and Benefits of UV and Tanning" held on the NIH Campus in September, 1998. My reading of this meeting was that a variety of uncertainties concerning, among other factors, the action spectra for cancer induction, make significant changes in regulatory efforts problematic. However, I understand that the FDA discourages the claim which has been made by some in the indoor tanning industry that tans induced by UVA are safer than tans induced by UVB. Good action spectra for a number of biological effects are required to answer some of the questions raised in the Workshop.

\section{Why are UVA Experiments Difficult ?}

Damage Cross Sections are Usually Small: Figure 1 and Figure 2 indicate that the action spectra for damaging DNA and inducing erythema both decrease monotonically throughout the UVA, reaching either low or undetectable levels near the visible. Thus, if experiments are performed with narrow spectral band ("monochromatic") light, effects are small and contamination by shorter wavelengths is a constant problem as is the need for proper "safe lights" during the conduct of experiments. Experiments in the UVB and UVC are technically less demanding, particularly for research groups whose primary interests are not spectroscopy or UV photobiology.

Conventional Light Sources are Limited in Intensity, Spectral Purity and/or Tunability: Lack of optimum light sources are a problem for photobiology, particularly in the UVA. Various lamps produce UVA, but spectra tend to be broad, so the exact wavelengths responsible for an effect is difficult to determine. Narrow band interference filters can reduce the spectral bandwidth to acceptable values and are inexpensive, but also reduce the flux reaching the target system to levels that are too low for experimental purposes. A few research groups use high pressure $\mathrm{Hg}$ or $\mathrm{Xe}$ arcs combined with either interference filters or grating monochromators. The Xe arc provides continuous wavelength coverage, but considerably lower intensity than that from a high pressure $\mathrm{Hg}$ arc at the wavelength of the Mercury emission lines. Even these higher fluxes are less than desirable. For example, a level of DNA damage that can be produced in about 30 seconds at 302 nm may require 30 minutes of irradiation at $365 \mathrm{~nm}$. Even worse, the intense UVA wavelengths available from a high pressure $\mathrm{Hg}$ arc are limited to 334 and $365 \mathrm{~nm}$.

\section{PHOTOBIOLOGY WITH AN FEL}

\section{The Ideal Light Source for UV Photobiology}

The ideal light source for photobiology would provide any wavelength over a range of intensities extending to very high values and would have a narrow spectral bandwidth (i.e., nearly monochromatic). Free electron lasers, and particularly the one planned for the Thomas Jefferson National Accelerator Facility, come closer to these requirements that any other source, particularly in the UVA where high intensity is critical. High time average power is especially important when the object to be irradiated is physically large, as in the case of animals - especially when multiple animals must be irradiated in parallel. 
The ideal source would also be easy to use, compact and inexpensive. Unfortunately, free electron lasers come nowhere close to meeting these requirements. While less than ideal in terms of cost and convenience, the outstanding operational characteristics of free electron lasers make them candidates for certain very important experiments that are simply undoable using any other light source.

\section{Research Objectives}

Many of the experiments that are required for better understanding of UVA photobiology can be classified as either determination of action spectra and kinetics, particularly the kinetics of repair. These should involve both photochemical and biological endpoints.

Photochemical Modifications of DNA: Studies of the photoproducts produced in DNA are important complements to experiments involving more biological endpoints. We need to know what photoproducts are being formed at different wavelengths and in what quantities. The development of new assays that use gel electrophoresis and repair enzymes that produce DNA chain breaks at oxidized bases will facilitate such measurements. Studies of DNA photoproducts usually do not need the same high intensities that will be needed for cancer induction experiments, so these are experiments that could be performed during the early phases of development of a source when irradiation levels have not reached their highest levels. Such experiments would provide valuable experience with a high intensity FEL source and guidance that would be useful in the design of more expensive animal studies.

Immunological Effects of UV: The discovery of UV-induced immunosuppression opens the possibility that UV can produce effects in organs that have not been directly exposed. For a review of this field see Noonan and De Fabo. ${ }^{11}$ The target for immunological effects has been proposed to be both DNA and urocanic acid, which is found in the stratum corneum. Action spectra can help answer this question, but have not been extended into the UVA because of the limitations of conventional sources. Extrapolations from UVB action spectra suggest that the UVA may also suppress the immune system. Immunosuppression, which may facilitate development of skin cancers, has been demonstrated in man, but is usually studied in a murine model. Experimental investigations of the immunological effects of ultraviolet radiation are facilitated by the ability to irradiate many animals simultaneously, which increases the power requirement of the radiation source.

Nonmelanoma Skin Cancers: The vast majority of human skin cancers are either basal cell carcinomas or squamous cell carcinomas. While far less dangerous than cutaneous malignant melanomas (vide infra), these are true cancers - they can metastasize if neglected - which arise from the epithelial cells that form the epidermis. Nonmelanoma skin cancers can be induced by ultraviolet radiation and are considered likely to increase significantly as a result of depletion of stratospheric ozone. Action spectra that generally parallel those for erythema and DNA damage have been determined in hairless mice, albeit by less than direct means, and becomes uncertain at longer UVA wavelengths. De Gruijl and Van der Leun have provided a comprehensive review of nonmelanoma skin cancers, from a biophysical perspective. ${ }^{7}$ of particular relevance to the present discussion is their comment regarding action spectra for induction of nonmelanoma skin cancers in animal models "Ideally one would like to irradiate the mice monochromatically - vary the wavelength in steps of $10 \mathrm{~nm}$ throughout the UV range, check the dose-time relationship at each wavelength, and ascertain how this relationship shifts as the effectiveness changes from one wavelength to the next. In reality this is near to impossible because ... the monochromator has to be powerful enough to be effective at each wavelength throughout the UV, and getting enough power (especially at $>340 \mathrm{~nm}$ ) may conflict with the demand of monochromaticity." With a high-powered FEL, it's possible!

Cutaneous Malignant Melanomas: While representing only a few percent of skin cancer incidence, these cancers are aggressively invasive and thus are responsible for most skin-cancer related mortality. These cancers are not restricted to the areas of the body exposed to direct sunlight, and are more prevalent in people who work indoors and are exposed to intense sunlight only intermittently (as when they are on vacation) than in people, such as farmers, who routinely work outside. Melanomas arise in melanocytes, which are the pigment producing cells of the skin, raising the possibility of the involvement of longer wavelengths. Clearly, determining action spectra for cutaneous malignant melanomas is one of the most important experimental goals in ultraviolet photobiology. However, measuring action spectra for cutaneous malignant melanomas in test animals is even more complicated than the corresponding experiments for nonmelanoma skin cancers, due to the lack of suitable animal models. Free electron lasers appear to offer the best ultraviolet source for pursuing such experiments as soon as appropriate test systems become available. 


\section{Source Requirements for UVA Photobiology}

High intensity is clearly one of the most important requirements for experiments involving the UVA, particularly if it is desirable to irradiate multiple animals simultaneously. A spectral band width of $1 \mathrm{~nm}$ will be more than adequate for most applications in UV photobiology. Thus spectral bandwidth will usually not be a limiting parameter if the source is any type of laser. Flexibility in wavelength selection is also critical. Note however, that wavelength scanning, as frequently required in spectroscopy, is not required. Experiments will usually be set-up for one wavelength for a given sample, and the wavelength changed no more frequently than the sample. Brightness, which implies good columnation is also important, because it may be necessary to distribute the radiation uniformly over a physically large sample or samples. Short-term stability of $\approx 5 \%$ should be adequate, provided facilities exist for monitoring the actual irradiance. In this case, integrated dose could be controlled to a far closer tolerance. Present protocols for experimental studies of UV induced skin cancers require repeated exposures - typically three exposures per week for several months. Thus, long-term availability is a far more important issue.

\section{Support Requirements for UVA Photobiology}

In the case of experiments involving in vitro irradiation of molecular or cellular samples, support requirements are modest. Some wet laboratory space and standard laboratory apparatus, a HEPA filtered hood and incubators for work with cells and standard facilities for measuring instantaneous and integrated radiation flux are the basic requirements. Support facilities for experiments involving animals are a far more significant issue, particularly for irradiation facilities located at institutions that lack ongoing animal research programs in other areas.

\section{Is Free Electron Laser Radiation a Valid Experimental Proxy for Sunlight?}

Free electron lasers represent an enormous advance in high-intensity, near-monochromatic sources of ultraviolet radiation. But are free electron lasers appropriate sources for experiments that attempt to mimic effects produced by sunlight. FEL radiation has several properties that are distinctly different from the radiation from the sun or most man-made sources of ultraviolet radiation to which biological organisms are exposed. These include the time structure, polarization and coherence of the radiation. High intensity linearly polarized light could easily have implications for photobiology because a fraction of the target molecules are at any instant not available as absorbers. In some biological systems, molecular reorientation is restricted so that during the course of an irradiation some potential target molecules may be effectively shielded. However, it is relatively easy to depolarize light in the UVA range, so polarization effects should not be a significant problem. Coherence is also unlikely to be a problem for photobiology, as the radiation will not usually be tightly focused, and, if necessary, optical elements can be used to destroy coherence.

The pulsing nature or "time structure" of the radiation does need some consideration. Some free electron lasers operate with a macro-bunch/micro-bunch time structure, which introduces the possibility that the average power - and hence heating effects - during a macro-bunch will be substantially greater than the long-term time-average power. However, the free electron laser most likely to be used for photobiology can generate a continuous beam of micro-pulses, without a superimposed macro-pulse structure (which is frequently referred to as a "CW" beam in the FEL community). Here the question of instantaneous heating is less of a problem that the simple question of whether delivering the radiation as a series of very short $(\approx \mathrm{ps})$ pulses at a high repetition rate $\left(\approx 10^{8} \mathrm{~Hz}\right)$ will significantly affect the resulting photobiology. At the "physiological" levels required in photobiology, this is unlikely to be a problem, because the photons are acting independently. However, the use of free electron lasers for photobiology must be verified by measuring a range of effects at the level of molecules, cells and whole organisms where the same dose can be delivered over the same time by both the FEL and a conventional source, to insure that the results are not distorted by the pulsing nature of an FEL.

\section{WHICH FEL?}

In the United States, there are three free electron lasers with ultraviolet capabilities that are operating or in some stage of construction or advanced planning. The OK4 at Duke University in Durham, NC, has been operating in the UV for over a year. The DUV FEL at Brookhaven National Laboratory, Upton, Long Island, NY, is nearing completion with commissioning scheduled to begin in the summer of 2000. Finally, plans are in place to upgrade the FEL presently operating in the infrared region at the Thomas Jefferson National Accelerator Facility in Newport News, VA, to provide ultraviolet radiation. Of these three free electron lasers, only the one at the Jefferson Facility is likely to achieve the very high power levels that will be required for UVA photobiology. Unfortunately, of the three emerging ultraviolet free electron lasers, only 
the one at Jefferson Lab is sited at an institution that does not already have the infrastructure for research animals, hence increasing the cost of performing critical experiments involving UVA radiation.

\section{ACKNOWLEDGMENTS}

Supported by the Office of Biological and Environmental Research of the United States Department of Energy.

\section{REFERENCES}

${ }^{1}$ Setlow, R. B. The Wavelengths in Sunlight Effective in Producing Skin Cancer: A Theoretical Analysis. Proc. Natl. Acad. Sci. U.S.A. 71, 3363-3366 (1974).

${ }^{2}$ Lindahl, T. and Wood, R. D. Quality control by DNA repair. Science 286, 1897-1905 (1999).

${ }^{3}$ Emrick, A. and Sutherland, J. C. Action Spectrum for Formation of Pyrimidine Dimers in T7 DNA: 180 to $365 \mathrm{~nm}$. Photochemistry and Photobiology $\underline{49}$, 35S- (1989).

${ }^{4}$ Freeman, S. E., Hacham, H., Gange, R. W., Maytum, D. J., Sutherland, J. C., and Sutherland, B. M. Wavelength Dependence of Pyrimidine Dimer formation in DNA of Human Skin Irradiated in situ. Proc. Natl. Acad. Sci., USA 86, 5605-5609 (1989).

${ }^{5}$ Gange, R. W., Park, Y.-K., Auletta, M., Kagetsu, N., Blackett, A. D., and Parrish, J. A. Action spectra for cutaneous responses to ultraviolet radiation, in The Biological Effects of UVA Radiation, F. Urbach and R.W. Gange, Editors, Praeger: New York. p. 57-65 (1986).

6Johns, H. E. Photoproducts Produced in Nucleic Acids by Ultraviolet Light. Radiation Research 733-755 (1966).

$7 \mathrm{de}$ Gruijl, F. R. and Van der Leun, J. C. Influence of ozone depletion on the incidence of skin cancer, in Environmental UV photobiology, A.R. Young, et al., Editors, Plenum: New Your. p. 89-112 (1993).

${ }^{8}$ Setlow, R. B., Grist, E., Thompson, K., and Woodhead, A. D. Wavelengths effective in induction of malignant melanoma. Proc. Natl. Acad. Sci. U. S. A. 90, 6666-6670 (1993).

${ }^{9}$ Berwick, M. Skin Cancers, in Multiple Primary' Cancers, A.I. Neugut, A.T. Meadows, and E. Robinson, Editors, Lippincott Williams and Wilkins: Philadelphia. p. 445-467 (1999).

$1^{10}$ Gasparro, F. P. and Berwick, M. Sunscreen use, wearing clothes, and number of nevi in 6- to 7-year-old European children. Journal of the National Cancer Institute 91, 1078-1080 (1999).

11 Noonan, R. P. and De Fabo, E. C. UV-induced imminosuppression, in Environmental UV photobiology, A.R. Young, et al., Editors, Plenum Press: New York. p. 113-148 (1993). 